\title{
Difference Sets of Null Density Subsets of $\mathbb{N}$
}

\author{
Dawoud Ahmadi Dastjerdi, Maryam Hosseini \\ Department of Mathematics, University of Guilan, Rasht, Iran \\ Email: ahmadi@guilan.ac.ir, maryamh2002@googlemail.com
}

Received December 29, 2011; revised February 9, 2012; accepted February 16, 2012

\section{ABSTRACT}

Let $\quad B \subset \mathbb{N}, \quad D^{1}(B):=B-B=\{a-b: a>b, a, b \in B\} \quad$ and for any $k \geq 2, \quad D^{k}(B)=D^{k-1}(B)-D^{k-1}(B)$. If $\bar{d}(B)=\limsup _{n \rightarrow \infty} \frac{|B \cap\{1, \cdots, n\}|}{n}$ is positive, then $B$ is considered as a large set with $\sum_{b \in B} \frac{1}{b}=\infty$. Its difference set $D^{1}(B)$ has both high density and rich structure. The set $A \subset \mathbb{N}$ with $\sum_{a \in A} \frac{1}{a}=\infty$ is also relatively large and it is a long standing conjecture that like sets with positive upper density they have arithmetic progression of arbitrary length. Here we show their difference set may not be substantial; for any $k \in \mathbb{N}$ there exists $A_{k} \subset \mathbb{N}$ such that $\sum_{a \in A_{k}} \frac{1}{a}=\infty$ and $d^{*}\left(D^{k-1}(A)\right)=0$.

Keywords: Difference Set; Density; $\Delta^{*}$-Set

\section{Introduction}

A subset $B$ of $\mathbb{N}$ has null density if

$$
d(B)=\lim _{n \rightarrow \infty} \frac{|B \cap\{1, \cdots, n\}|}{n}=0 .
$$

Perhaps, the most prominent general result in this case is a theorem conjectured by Erdős and proved by Rusza [1]. It states that if $d(B)=0$ then

$$
\lim _{n \rightarrow \infty} \frac{\left|D^{1}(B) \cap\{1, \cdots, n\}\right|}{|B \cap\{1, \cdots, n\}|}=\infty
$$

where $D^{1}(B):=B-B=\{a-b: a>b, a, b \in B\}$.

Our concern here is to consider the null density subsets in the family $\mathcal{F}=\left\{A \subset \mathbb{N}: \sum_{a \in A} \frac{1}{a}=\infty\right\}$. These sets can be considered "large" sets among null density sets. To have a comparison with sets of higher densities, note that if $A \in \mathcal{F}$ and $n \in \mathbb{N}, A-n=\{a-n: a \in A\} \in \mathcal{F}$ and $\mathcal{F}$ has Ramsey property, that is, if $A \in \mathcal{F}$ and $A$ is partitioned into finitely many sets then at least one of the elements of the partition lies in $\mathcal{F}$. These two properties, being invariant under translation and having Ramsey property, hold for

$$
\mathcal{D}=\left\{B \subset \mathbb{N}: \bar{d}(B)=\limsup _{n \rightarrow \infty} \frac{|A \cap\{1, \cdots, n\}|}{n}>0\right\}
$$

as well. However, if $B \in \mathcal{D}$ then $D^{1}(B)$ is a $\Delta^{*}$-set [2]. This means $D^{1}(B)$ is both large and structured set. For instance, it is syndetic: there exists a $p$ such that $[n, n+p] \cap D^{1}(B) \neq \varnothing$ for any $n$ in $\mathbb{N}$. By definition, a $\Delta^{*}$-set intersects the difference set of any infinite natural sequence and amongst many structures it is $I P$ which means there is a sequence of natural numbers $\left\{b_{n}\right\}_{n \in \mathbb{N}}$ such that all of its finite sums are in $B$ [3].

From largeness point of view, $\mathcal{F}$ may be considered next to $\mathcal{D}$ for $\mathcal{D} \subset \mathcal{F}$ [4]. Note that there is also a family

$$
\mathcal{D}^{*}=\left\{A \subset \mathbb{N}: d^{*}(A)=\limsup _{m-n \rightarrow \infty} \frac{|A \cap\{m, \cdots, n\}|}{m-n}>0\right\}
$$

which $d^{*}(A)$ is called the upper Banach density of $A$. We have that $\mathcal{D} \subset \mathcal{D}^{*}$ and $\mathcal{D}^{*}$ is invariant under translation and satisfies Ramsey property [5]. Also, if $B \subset \mathcal{D}^{*}$ then $D^{1}(B)$ is $\Delta^{*}[5]$ and $B$ has arithmetic progression of arbitrary length [6]. However, $B$ may not be $\mathcal{F}$-large. For instance, $B=\left\{2^{n}, 2^{n}+1, \cdots, 2^{n}+n: n \in \mathbb{N}\right\} \in \mathcal{D}^{*}$ but $\sum_{b \in B} \frac{1}{b}<\infty$.

Erdös conjectured that elements of $\mathcal{F}$ have arithmetic progression of arbitrary length [7]. An important subset of natural numbers, which is the set of prime numbers lies in $\mathcal{F}$ and the conjecture was proved positively by 
Green-Tao [8].

In order to present a substantial contrast of sets in $\mathcal{F}$ with those in $\mathcal{D}$, we will show that for any $k \in \mathbb{N}$ there is $A \in \mathcal{F}$ such that $D^{k}(A)=D\left(D^{k-1}(A)\right)$; the $k$ th difference set of $A$ even does not lie in $\mathcal{D}^{*}$.

Throughout this note, unless otherwise stated, by an interval we mean an interval of integers. So for instance, $[a, b)=\{x \in \mathbb{Z}: a \leq x<b\}$.

\section{Reference Set}

Here we introduce and investigate the properties of a subset of non-negative integers suitably defined for our later use.

Let $Y=\left\{y_{0}=0, y_{1}, y_{2}, \cdots\right\}$ be a set of non-negative integers with the property that $y_{n} \in \mathbb{N}, y_{n+1}>2 y_{n}$ for any $n \geq 1$. For any $n \in \mathbb{N}$ let $l_{n}$ be the largest integer strictly less than $\frac{y_{n+1}}{2 y_{n}}$. Set $c_{k_{1}, \cdots, k_{n}}:=2 k_{1} y_{1}+2 k_{2} y_{2}+\cdots+2 k_{n} y_{n}$ for all those $0 \leq k_{i} \leq l_{i}, \quad i=1, \cdots, n$ such that $2 k_{1} y_{1}+2 k_{2} y_{2}+\cdots+2 k_{n} y_{n} \in\left[y_{0}, y_{n+1}\right)$. Also set

$$
d_{k_{1}, \cdots, k_{n}}:= \begin{cases}c_{k_{1}, \cdots, k_{n}}+y_{1}, & \text { if } c_{k_{1}, \cdots, k_{n}}+y_{1} \leq y_{n+1} \\ y_{n+1}, & \text { otherwise. }\end{cases}
$$

Definition 1.1. Let $Y$ be as above. The set of nonnegative integers

$$
R_{Y}=\bigcup_{n=1}^{\infty}\left(\bigcup_{k_{1}=0 k_{2}=0}^{l_{1}} \ldots \bigcup_{k_{n}=0}^{l_{2}}\left(\left[c_{k_{1}, \cdots, k_{n}}, d_{k_{1}, \cdots, k_{n}}\right) \cap\left[y_{0}, y_{n}\right)\right)\right)
$$

is called the reference set associated to $Y$.

The reference set can also be seen as follows. Set $\Omega_{n}:=\left[y_{0}, y_{n}\right) \cap R_{Y}$, then

$$
\begin{aligned}
\Omega_{1}= & {\left[0, y_{1}\right), } \\
\Omega_{2}= & \left(\Omega_{1} \cup \cdots \cup\left(2 k_{1} y_{1}+\Omega_{1}\right) \cup\right. \\
& \left.\cdots \cup\left(2 l_{1} y_{1}+\Omega_{1}\right)\right) \cap\left[0, y_{2}\right), \\
\Omega_{3}= & \left(\Omega_{2} \cup \cdots \cup\left(2 k_{2} y_{2}+\Omega_{2}\right) \cup\right. \\
& \left.\cdots \cup\left(2 l_{2} y_{2}+\Omega_{2}\right)\right) \cap\left[0, y_{3}\right), \\
\vdots & \\
\Omega_{n+1}= & \left(\Omega_{n} \cup \cdots \cup\left(2 k_{n} y_{n}+\Omega_{n}\right) \cup\right. \\
& \left.\cdots \cup\left(2 l_{n} y_{n}+\Omega_{n}\right)\right) \cap\left[0, y_{n}\right)
\end{aligned}
$$

where $2 k_{i} y_{i}+\Omega_{i}=\left\{2 k_{i} y_{i}+x: x \in \Omega_{i}\right\}$.

Let us now examine when $R_{Y} \backslash\{0\}$ for a given $Y$ belongs to $\mathcal{F}\left(=\left\{A \subset \mathbb{N}: \sum_{a \in A} \frac{1}{a}=\infty\right\}\right)$.

Lemma 1.2. Suppose $Y=\left\{y_{0}=0, y_{1}, y_{2}, \cdots\right\} \subset \tilde{\mathbb{N}}$ with $y_{n+1}>2 y_{n}$ and let $R_{Y}$ be its associated reference set. Suppose for sufficiently large $n$,

$$
\frac{y_{n+2}}{y_{n+1}}>\left(\frac{y_{n+1}}{y_{n}}\right)^{2+\varepsilon},
$$

for some $\varepsilon>0$. Then $R_{Y} \backslash\{0\} \in \mathcal{F}$.

Proof. First note that (2) implies

$$
\lim _{n \rightarrow \infty} \frac{y_{n+1}}{y_{n}}=\infty ;
$$

and also if we set

$$
\alpha_{n}:=\frac{\ln \left(y_{n+2}\right)-\ln \left(y_{n+1}\right)}{2\left(\ln \left(y_{n+1}\right)-\ln \left(y_{n}\right)\right)}
$$

then $\liminf _{n \rightarrow \infty} \alpha_{n}>1$.

Now let for $n \geq 1, I_{n}:=\operatorname{card}\left(\left[y_{n-1}, y_{n}\right] \cap R_{Y}\right)$. Then

$$
\begin{aligned}
& \frac{y_{n}-2 y_{n-1}}{2 y_{n-1}}\left(\operatorname{card}\left(R_{Y} \cap\left[0, y_{n-1}\right)\right)\right) \leq I_{n} \\
& \leq \frac{y_{n}-y_{n-1}}{2 y_{n-1}}\left(\operatorname{card}\left(R_{Y} \cap\left[0, y_{n-1}\right)\right)\right) .
\end{aligned}
$$

But for $0 \leq k_{n}<l_{n}$,

$$
\begin{aligned}
\operatorname{card}\left(R_{Y} \cap\left[0, y_{n}\right)\right) & =\operatorname{card}\left(R_{Y} \cap\left[2 k_{n} y_{n},\left(2 k_{n}+1\right) y_{n}\right)\right) \\
& =\left(I_{1}+I_{2}+\cdots+I_{n}\right) .
\end{aligned}
$$

So by estimating any $\frac{1}{r}, r \in\left[2 k_{n} y_{n},\left(2 k_{n}+1\right) y_{n}\right)$ with $\frac{1}{\left(2 k_{n}+1\right) y_{n}}$,

$$
\sum_{r \in R_{Y} \cap\left(0, y_{m+1}\right)} \frac{1}{r}>\sum_{n=1}^{m} e_{n}
$$

where

$$
e_{n}:=\left(I_{1}+\cdots+I_{n}\right)\left(1 / y_{n}+1 / 3 y_{n}+\cdots+1 /\left(y_{n+1}-3 y_{n}\right)\right) .
$$

Note that by (3) for sufficiently large $n$ we have $y_{n+1}-3 y_{n}>0$. Now

$$
\begin{aligned}
\frac{e_{n+1}}{e_{n}}= & \frac{I_{1}+\cdots+I_{n+1}}{I_{1}+\cdots+I_{n}} \times \frac{\frac{1}{y_{n+1}}+\frac{1}{3 y_{n+1}}+\cdots+\frac{1}{y_{n+2}-3 y_{n+1}}}{\frac{1}{y_{n}}+\frac{1}{3 y_{n}}+\cdots+\frac{1}{y_{n+1}-3 y_{n}}} \\
& =\left(1+\frac{I_{n+1}}{I_{1}+\cdots+I_{n}}\right) \times \frac{\frac{1}{y_{n+1}}+\frac{1}{3 y_{n+1}}+\cdots+\frac{1}{y_{n+2}-3 y_{n+1}}}{\frac{1}{y_{n}}+\frac{1}{3 y_{n}}+\cdots+\frac{1}{y_{n+1}-3 y_{n}}} .
\end{aligned}
$$

Also by (5),

$$
I_{n+1} \geq\left(\frac{y_{n+1}-2 y_{n}}{2 y_{n}}\right)\left(I_{1}+\cdots+I_{n}\right) .
$$

Therefore, 


$$
\begin{aligned}
\frac{e_{n+1}}{e_{n}} \geq & \left(1+\frac{y_{n+1}-2 y_{n}}{2 y_{n}}\right) \times \frac{y_{n}}{y_{n+1}} \times \frac{\int_{1}^{\frac{y_{n+2}-3 y_{n+1}}{y_{n+1}}} \frac{1}{2 x-1} \mathrm{~d} x}{1+\int_{1}^{\frac{y_{n+1}-3 y_{n}}{y_{n}}} \frac{1}{2 x-1} \mathrm{~d} x} \\
& =\frac{1}{2} \times \frac{\int_{1}^{\frac{y_{n+2}-3 y_{n+1}}{y_{n+1}}} \frac{1}{2 x-1} \mathrm{~d} x}{1+\int_{1}^{\frac{y_{n+1}-3 y_{n}}{y_{n}}} \frac{1}{2 x-1} \mathrm{~d} x} .
\end{aligned}
$$

By considering (3) this implies

$$
\begin{aligned}
\liminf _{n \rightarrow \infty} \frac{e_{n+1}}{e_{n}} & \geq \liminf _{n \rightarrow \infty}\left[\frac{1}{2} \times \frac{\ln \left(\frac{y_{n+2}}{y_{n+1}}\right)}{\ln \left(\frac{y_{n+1}}{y_{n}}\right)}\right] \\
& =\liminf _{n \rightarrow \infty} \alpha_{n}>1 .
\end{aligned}
$$

Now the proof is complete by the ratio test.

Let $b \geq 2$, then an example for $Y$ satisfying the above lemma is

$$
Y=\left\{0,2 b^{(2 b)}, \cdots,(2 b)^{(2 b)^{n}}, \cdots\right\} .
$$

For this example $\liminf _{n \rightarrow \infty} \alpha_{n}=\lim _{n \rightarrow \infty} \alpha_{n}=b$.

Remark 1.3. Similar arguments as the proof of the above lemma shows that if either (3) does not hold or if (3) holds but there is some $\varepsilon>0$ such that for infinitely many $n$,

$$
\frac{y_{n+2}}{y_{n+1}}<\left(\frac{y_{n+1}}{y_{n}}\right)^{2-\varepsilon}
$$

then $\sum_{r \in R_{Y}} \frac{1}{r}<\infty$. We give a sketch of proof for the latter.

Suppose $\alpha_{n}$ is defined as (4). Then by (8), we have $\limsup _{n \rightarrow \infty} \alpha_{n}<1$. Let $I_{i}$ be as above and set

$e_{n}:=\left(I_{1}+\cdots+I_{n}\right)\left(\frac{1}{2 y_{n}}+\frac{1}{4 y_{n}}+\cdots+\frac{1}{2 l_{n} y_{n}}\right)$. Using the left inequality in (5), we have

$$
\sum_{r \in R_{Y} \cap\left(0, y_{m}\right)} \frac{1}{r} \leq I_{1}+\sum_{n=1}^{m} e_{n} .
$$

Now

$$
\frac{e_{n+1}}{e_{n}} \leq \frac{y_{n+1}+y_{n}}{2 y_{n+1}} \times \frac{1+\int_{2}^{l_{n+1}} \frac{1}{x} \mathrm{~d} x}{\int_{2}^{l_{n}} \frac{1}{x} \mathrm{~d} x}
$$

From (3), we have $\int_{2}^{l_{n}} \frac{1}{x} \rightarrow \infty$. This and the fact that $\frac{y_{n}}{y_{n+1}} \rightarrow 0$ implies

$$
\limsup _{n \rightarrow \infty} \frac{e_{n+1}}{e_{n}} \leq \limsup _{n \rightarrow \infty} \alpha_{n}<1
$$

and we are done.

\section{Main Result}

We will show that comparing to a set of positive upper Banach density, the difference set, $D(A)=A-A$ of a set $A$ with $\sum_{a \in A} \frac{1}{a}=\infty$ and $d^{*}(A)=0$ can be very sparse. The next theorem gives a class of examples with this property.

Theorem 2.1. Let $k \in \mathbb{N}$, then there exists $A_{k} \subset \mathbb{N}$ with $\sum_{a \in A_{k}} \frac{1}{a}=\infty$ and $D^{k}\left(A_{k}\right) \subset A_{0}$ such that $A_{0}$ is not $\Delta^{*}$. In particular, $d^{*}\left(D^{k-1}\left(A_{k}\right)\right)=0$.

Proof. Let $b \geq 2, y_{0}=0, y_{1}=2^{k+1} b, \quad y_{n}=y_{1}^{y_{1}^{n-1}}$ and $Y=\left\{y_{0}, y_{1}, y_{2}, \cdots\right\}$. Consider the following symmetric subsets of integers:

$$
\begin{gathered}
F_{0}^{j}=\{0\}, \\
F_{i-1}^{j}=\left\{\frac{-y_{i}}{2^{j+2} y_{i-1}}+1, \cdots,-1,0,1, \cdots, \frac{y_{i}}{2^{j+2} y_{i-1}}-1\right\}
\end{gathered}
$$

for $0 \leq j \leq k$ and $i \geq 2$. Set

$$
A_{j}:=\bigcup_{n=0}^{\infty}\left(\bigcup_{a_{0} \in F_{0}^{j}} \bigcup_{a_{1} \in F_{1}^{j}} \cdots \bigcup_{a_{n} \in F_{n}^{j}} I_{a}^{j}\right)
$$

where $\boldsymbol{a}:=a_{0}, a_{1}, \cdots a_{n}$ and $I_{a_{0}, a_{1}, \cdots, a_{n}}^{j}$ is the interval

$$
\begin{aligned}
& {\left[2 a_{0} y_{0}+2 a_{1} y_{1}+\cdots+2 a_{n} y_{n}-\frac{y_{1}}{2^{j+1}},\right.} \\
& \left.2 a_{0} y_{0}+2 a_{1} y_{1}+\cdots+2 a_{n} y_{n}+\frac{y_{1}}{2^{j+1}}\right)
\end{aligned}
$$

in $\mathbb{N}$. Hence $A_{j+1} \subset A_{j}$ and $A_{j}$ is a union of subintervals of non-zero integers with the first subinterval of length $\frac{y_{1}}{2^{j+1}}$ and all others of length $\frac{y_{1}}{2^{j}}$. To have a picture of $A_{j}$ 's consider $A_{0}$ which is a prototype for others. Then $A_{0}$ may be considered as $\bigcup_{n=1}^{\infty} A_{0}^{n}$ in which

$$
\begin{gathered}
A_{0}^{1}=A_{0} \cap\left[0, y_{1}\right)=\left[0, \frac{y_{1}}{2}\right]=R_{Y} \cap\left[y_{0}, \frac{y_{1}}{2}\right)-\frac{y_{0}}{2}, \\
A_{0}^{2}=A_{0} \cap\left[y_{1}, y_{2}\right)=R_{Y} \cap\left[y_{1}, \frac{y_{2}}{2}\right]-\frac{y_{0}}{2}-\frac{y_{1}}{2},
\end{gathered}
$$

$$
A_{0}^{n+1}=A_{0} \cap\left[y_{n}, y_{n+1}\right)=R_{Y} \cap\left[y_{n}, \frac{y_{n+1}}{2}\right)-\sum_{i=1}^{n} \frac{y_{i}}{2}
$$


That is, $A_{0}$ has as half elements as $R_{Y}$ in $\left[0, y_{n+1}\right)$ which are shifted to the left appropriately. So

$$
2 \sum_{a \in A_{0} \cap\left(0, y_{n+1}\right)} \frac{1}{a}>\sum_{r \in R_{Y}} \cap\left(0, y_{n+1}\right) \frac{1}{r} .
$$

By Lemma 1.2, one obtains that $\sum_{r \in R_{Y} \backslash\{0\}} \frac{1}{r}$ and hence $\sum_{a \in A_{0} \backslash\{0\}} \frac{1}{a}=\infty$. (Later we will prove that $\sum_{a \in A_{k}} \frac{1}{a}=\infty$. So divergence of $\sum_{a \in A_{0} \backslash\{0\}} \frac{1}{a}$ will be a consequence of that as well).

Now we claim that $A_{0}$ is not $\Delta^{*}$. First note that $\left\{y_{n+1}-y_{i}: 1 \leq i \leq n\right\} \subseteq\left[y_{n+1}-y_{n}, y_{n+1}\right]$. Then the claim is established by noting that by the above definition for $A_{0}$

$$
A_{0} \cap\left[y_{n+1}-y_{n}, y_{n+1}\right]=\varnothing
$$

which implies $A_{0}$ does not intersect $D^{1}(Y \backslash\{0\})=\left\{y_{i}-y_{j}: i>j>1\right\}$ and so it is not $\Delta^{*}$.

The sets $F_{i-1}^{k}$ 's and $A_{j}$ 's are defined in such a way that $D^{1}\left(A_{j}\right) \subseteq A_{j-1}$, for $1 \leq j \leq k$. To see this, suppose $x$ and $y$ are two elements of $A_{j}$ and $x>y$. Then there exists $n \in \mathbb{N}$ such that

$$
\begin{aligned}
& 2 a_{0} y_{0}+2 a_{1} y_{1}+\cdots+2 a_{n} y_{n}-\frac{y_{1}}{2^{j+1}} \\
& \leq x<2 a_{0} y_{0}+2 a_{1} y_{1}+\cdots+2 a_{n} y_{n}+\frac{y_{1}}{2^{j+1}}
\end{aligned}
$$

and also $m \leq n$ with

$$
\begin{aligned}
& 2 b_{0} y_{0}+2 b_{1} y_{1}+\cdots+2 b_{m} y_{m}-\frac{y_{1}}{2^{j+1}} \\
& \leq y<2 b_{0} y_{0}+2 b_{1} y_{1}+\cdots+2 b_{m} y_{m}+\frac{y_{1}}{2^{j+1}}
\end{aligned}
$$

where $a_{i}, b_{i} \in F_{i-1}^{j}$. So $a_{i}$ and $b_{i}$ are in $\left[\frac{-y_{i}}{2^{j+2} y_{i-1}}+1, \frac{y_{i}}{2^{j+2} y_{i-1}}-1\right]$ or equivalently,

$$
\frac{-y_{i}}{2^{j+1} y_{i-1}}+2 \leq a_{i}-b_{i} \leq \frac{y_{i}}{2^{j+1} y_{i-1}}-2 \text {. }
$$

Therefore, $x-y \in A_{j-1}$.

To complete the proof, it remains to show that for each $A_{j}, \quad \sum_{a \in A_{j} \backslash\{0\}} \frac{1}{a}=\infty$. We already have proved this fact for $A_{0}$ and we will prove that for $A_{k}$ and since $A_{k} \subset A_{j}$, $0 \leq j \leq k-1$ we are done. So consider $F_{i}^{k}$ in (9) and let

$$
\begin{aligned}
& {F_{0}^{\prime k}}^{\prime}:=\{0\}=F_{0}^{k}, \\
& F_{i}^{\prime k}:=F_{i}^{k}+\frac{y_{i+1}}{2^{k+2} y_{i}}-1=\left\{0,1, \cdots, \frac{y_{i+1}}{2^{k+1} y_{i-1}}-2\right\}, \forall i \geq 1 .
\end{aligned}
$$

Also set

$$
\begin{aligned}
A_{k}^{\prime}:= & \bigcup_{n=0}^{\infty} \bigcup_{b_{0} \in F_{0}^{\prime j}} \cdots \bigcup_{b_{n} \in F_{n}^{\prime k}}\left(2 b_{0} y_{0}+\cdots+2 b_{n} y_{n},\right. \\
& \left.2 b_{0} y_{0}+\cdots+2 b_{n} y_{n}+\frac{y_{1}}{2^{k}}\right) .
\end{aligned}
$$

Then $\sum_{a \in A_{k}} \frac{1}{a} \geq \sum_{b \in A_{k}^{\prime}} \frac{1}{b}$. Now using the same arguments as in Lemma 1.2, we prove that $\sum_{b \in A_{k}^{\prime}} \frac{1}{b}$ is divergent.

If we let

$$
\begin{aligned}
& I_{1}^{\prime}:=\operatorname{card}\left(\left[0, y_{1}\right) \cap A_{k}^{\prime}\right), \cdots, \\
& I_{n}^{\prime}:=\operatorname{card}\left(\left[y_{n-1}, y_{n}\right) \cap A_{k}^{\prime}\right), \cdots,
\end{aligned}
$$

then

$$
\begin{aligned}
& I_{1}^{\prime}=\frac{1}{2^{k}} y_{1}, \cdots \\
& I_{n}^{\prime} \geq \frac{1}{2^{k}}\left(\frac{y_{n}-2 y_{n-1}}{2 y_{n-1}}-2\right)\left(I_{1}^{\prime}+\cdots+I_{n-1}^{\prime}\right), \cdots
\end{aligned}
$$

Let $l_{n}$ be as in the definition of $R_{Y}$; then

$$
\sum_{b \in A_{k}^{\prime} \backslash\{0\}} \frac{1}{b} \geq \sum_{n \in \mathbb{N}} e_{n}^{\prime}
$$

where

$$
\begin{aligned}
e_{n}^{\prime} & :=\left(I_{1}^{\prime}+\cdots+I_{n}^{\prime}\right)\left(\frac{1}{y_{n}}+\frac{1}{3 y_{n}}+\cdots+\frac{1}{2^{-k}\left(2 l_{n}-3\right) y_{n}}\right) \\
& =\left(I_{1}^{\prime}+\cdots+I_{n}^{\prime}\right)\left(\frac{1}{y_{n}}+\frac{1}{3 y_{n}}+\cdots+\frac{1}{2^{-k}\left(y_{n+1}-5 y_{n}\right)}\right) .
\end{aligned}
$$

So

$$
\begin{aligned}
\liminf _{n \rightarrow \infty} \frac{e_{n+1}^{\prime}}{e_{n}^{\prime}} \geq & \lim _{n \rightarrow \infty} \frac{I_{1}^{\prime}+\cdots+I_{n+1}^{\prime}}{I_{1}^{\prime}+\cdots+I_{n}^{\prime}} \\
& \times \frac{\frac{1}{y_{n+1}}+\cdots+\frac{1}{2^{-k}\left(y_{n+2}-5 y_{n+1}\right)}}{y_{n}+\cdots+\frac{1}{2^{-k}\left(y_{n+1}-5 y_{n}\right)}} \\
\geq & \liminf _{n \rightarrow \infty}\left(\frac{1}{2^{k+1}} \times \frac{\int_{2}^{\frac{y_{n+2}-5 y_{n+1}}{2^{k} y_{n+1}}} \frac{1}{2 x-1} \mathrm{~d} x}{\left.1+\int_{1}^{\frac{y_{n+1}-5 y_{n}}{2^{k} y_{n}}} \frac{1}{2 x-1} \mathrm{~d} x\right)}\right. \\
= & \frac{1}{2^{k+1}} \times \liminf _{n \rightarrow \infty} \frac{y_{1}^{n+2}-y_{1}^{n+1}}{y_{1}^{n+1}-y_{1}^{n}}=\frac{y_{1}}{2^{k+1}}>1 .
\end{aligned}
$$

Let $A_{k}$ be the set defined in the proof of the above theorem and let $S \subset \mathbb{N}$ be a syndetic set with the larg- 
est gap smaller than $b$. Then the conclusion of the above theorem applies for $A_{k} \cap S$. This conclusion also holds if we let $Y=\left\{y_{0}=0, y_{1}, y_{2}, \cdots\right\}$ with $y_{1}=e^{k+1} b_{1}$, $y_{n}=b_{n} y_{1}^{y_{1}^{p(n)}}, 2 \leq b_{1} \leq \cdots \leq b_{n} \leq b_{n+1} \leq \cdots$ where $e$ is an even integer and $p(n)$ is an increasing integer valued function with $p(1) \geq 1$.

Also recall that the theorem of Rusza [1] for sets of null density states that the difference set is considerablely larger than the set itself, that is, if $d(B)=0$ then (1) holds. However, in examples such as those in the above theorem, one tries to have a difference set which is as small as possible. Our approach was to have the arithmetic progression of long possible lengths. Therefore, such examples not only do not contradict the Erdös conjecture, but strongly are in the favor of it.

\section{REFERENCES}

[1] I. Z. Rusza, "On Difference-Sequences," Acta Arithmetica, Vol. 25, 1974, pp. 151-157.

[2] V. Bergelson and N. Hindman, "Additive and Multiplicative Ramsey Theorems in $\mathbb{N}$-Some Elementary Results,"
Combinatorics, Probability and Computing, Vol. 2, 1993, pp. 221-241. doi:10.1017/S0963548300000638

[3] V. Bergelson, "Partition Regular Structures Contained in Large Sets Are Abundant," Journal of Combinatorial Theory, Series A, Vol. 93, No. 1, 2001, pp. 18-36. doi:10.1006/jcta.2000.3061

[4] T. C. Brown and A. R. Freedman, "Arithmetic Progressions in Lacunary Sets," Rocky Mountain Journal of Mathematics, Vol. 17, No. 3, 1987, pp. 587-596. doi:10.1216/RMJ-1987-17-3-587

[5] V. Bergelson, N. Hindman and R. McCutchen, "Notions of Size and Combinatorial Properties of Quotient Sets in Semigroups," Topology Proceedings, Vol. 23, 1998, pp. 23-60.

[6] E. Szemerdi, "On Sets of Integers Containing No $k$ Elements in Arithmetic Progression," Acta Arithmetica, Vol. 27, 1975, pp. 199-245.

[7] P. Erdös, "Problems and Results in Combinatorial Number Theory," Astrisque, 1975, pp. 295-310.

[8] B. Green and T. Tao, "The Primes Contain Arbitrarily Long Arithmetic Progressions," Annals of Mathematics, Vol. 167, No. 2, 2004, pp. 481-547. doi:10.4007/annals.2008.167.481 